\section{The driving force of pacemakers}

MARGARET REICH

Senior Staff Editor

Even General Motors would have to agree: Any generator that produces enough energy to pump 2,100 gallons of blood through a 60,000 -mile circulatory system deserves to be called the "Heartbeat of America."

The natural pacemaker or sinus node serves as the driving force behind the heart's pumping action. To understand the pacemaker's role in this delivery of nutrient-rich blood throughout the body, let's examine how the healthy heart operates.

\section{Normal heart function}

The left and right atria and ventricles, which are the chambers that compose the heart, are central to this pumping muscle. Incoming blood that has already made the trip throughout the body is full of carbon dioxide. Veins carry the deoxygenated blood to the right atrium.

Next stop is the right ventricle. Here the blood is carried to the lungs by way of the pulmonary artery. Once in the lungs, the blood receives oxygen and travels to the left atrium and then on to the left ventricle. Major pumping action occurs here. The left ventricle pumps approximately 3 ounces of oxygen-fresh blood per beat. It's distributed from the aortic valve, the valve just over the heart, to the rest of the body via arteries and capillaries. After the trip is completed, veins carry the "used" blood back to the right atrium where the cycle begins again.

\section{Cardiac rhythm}

The number of beats of normal cardiac rhythm is maintained by the sinus node in the heart. The sinus node or natural pacemaker receives electric signals from the brain and spinal cord (sympathetic nervous system). Hormones released by the adrenal and thyroid glands also influence the sinus node. These electric impulses are received by the atrial conducting fibers.
The signals slowly proceed to stimulate cells found in the " $A-V$ " node, the area between the atria, just before the entrance to the ventricles. The electronic impulses speed up considerably as they prepare to stimulate the ventricular conducting fibers. These fibers excite the left ventricle, causing the heart to contract.

Because the heart beats on demand, cardiac rate varies from person to person. The average adult's heart contracts 72 to 78 beats per minute (BPM) during nonstressful periods, or as high as 200 BPM during exercise. An infant experiences 110 to 150 BPM.

\section{Abnormal heart rhythms}

Everyone experiences skipped beats or palpitations, especially during excitement or stress. Problems arise, however, when such occasional irregularities (arrhythmias) become commonplace.

\section{Bradycardia}

This cardiac arrhythmia, or abnormal heart beat, occurs when the heart rate falls below 60 BPM. Usually occurring at rest, bradycardia can be found in young, healthy athletes with no existing cardiac problems. But, the elderly are more prone to such irregularities due to pre-existing cardiac disease such as hardening of the arteries (arteriosclerosis).

Medication prescribed for a pre-exisitng cardiac problem can cause bradycardia. Sometimes, a genetic heart defect may be responsible for the abnormal rhythm.

Whatever the cause, the bradycardiac person frequently will not recognize the slow heart beat during rest. It's usually when he or she exercises that a lack of stamina seems to be evident.

If you are experiencing such symptoms and your pulse rate falls below 40 BPM, your doctor may prescribe medicine such as atropine to increase the heart rate.

\section{Tachycardia}

Just the opposite of bradycardia, tachycardia is a rapid pulse rate, usually 100 BPM or more. In this instance, the electric impulses from the sympathetic nervous system come at a rapid pace. 


\section{Help is just a phone call away}

Patients can now have their pacemakers checked over the phone. By placing the phone receiver over the transmitting device accompaning all pacemakers, the wearer can have his or her generator monitored telemetrically. On the other end of the line, the monitoring service prints out an ECG and forwards it on to the patient's physician within a day.

New pacemaker recipients call in twice a week during the first 6 weeks. Thereafter most are monitored twice a month. The fee for this service is about $\$ 40$ for each call, some of which is usually paid for in part by insurance or Medicare.

One such service, Pulseline, also answers questions patients may have concerning any cardiac problem.

For more information, write: Pulseline, PO Box 54305, Atlanta, GA 30308 or call: 1-800241-6993.

While a quick pulse is normal during exercise, sometimes noncardiac causes such as anemia, bleeding, infections and high body temperature may result in tachycardia. Similarly, nicotine, caffeine, and marijuana increase the heart rate.

Tachycardia may also accompany other cardiac conditions, such as inflammation of the heart's muscular walls.

As with bradycardia, symptoms are usually nonexistent. Sometimes, though, persons with this conditon may actually be aware of a faster heart pace with stronger contractions.

If the heart is beating very rapidly, sensations of dizziness, shortness of breath, or chest pain may occur. There are many types of tachycardias. Some may require drug treatment and some may not. Some of the medications that are used for the various types are quinidine, procainamide, beta-blockers, and calcium-channel blockers. All of the medications will correct an abnormal rhythm to a normal one, or slow the heart rate.

When tachycardia can be traced to an existing problem, treating that initial condition usually resolves the tachycardia.

Before diagnosing and treating any arrhythmia, your physician will perform an electrocardiogram (ECG). This procedure indicates how well your heart is working by measuring the small electric currents created by the expansion and contraction cycles.

Often an ECG is performed in conjunction with a stress test-bicycle or treadmill exercises - to see how well your heart performs under extra strain. Your physician may suggest a pacemaker only after such testing and other failed-treatments warrant this approach.

\section{Artificial pacemakers}

History

The first artificial pacemaker was patented in 1933 but a battery-operated external device did not actually become available until 1958 . Invented by Paul M. Zoll, MD, the external pacemaker still proves useful as a temporary mechanism in emergencies. It's quick and easy to hook up with minimal risk of infection.

\section{Internal pacemakers}

By 1960 , a completely implantable unit was developed. Since then, substantial technologic changes have made the device lighter (less than 1-1/ $2 \mathrm{oz}$ ), smaller (about the size of a silver dollar), and more efficient.

Three basic parts - two of which are implanted generally comprise modern pacemakers. A lithiumiodide battery commonly serves as the power source. Depending on the type of generator, the battery can last 8 to 12 years. Integrated microchips control the sensing and pacing functions.

Both mechanisms are encased in titanium. This metallic element is compatible with the body's chemical makeup. The case is air- and water-tight against the body's own fluids.

Flexible plastic tubes connect the generator to the pacing leads. Corrosion-resistant wire leads, small in diameter, are insulated with urethane. One end of the lead connects to the pacemaker while the other platinum electrode reaches the heart muscle.

Finally, the radio-frequency programmer allows the physician to adjust the pacemaker rate from outside the body. 


\section{Types of internal pacemakers}

Overall, pacemakers can be classified as single- or dual-chambered. The lead in a single-chamber generator is usually placed in the ventricle. Sensing and pacing of electrical impulses occurs in the ventricle only. Atrial contractions are not regulated.

On the other hand, the dual chamber places leads in both the right atrium and ventricle. It senses the incoming electric impulses in the atrium, regulates the contractions, and controls ventricular pumping action. The shortened 4- to 5-year battery life is due to the extra energy demands required of dual pacing devices.

Both single- and dual-chambered pacemakers are implanted without surgically opening the chest cavity. Leads are threaded through the cephalic vein in the neck. The generator itself is sewn under the skin in the shoulder or abdomen.

\section{Generator rates}

Within these two broad categories, pacemakers may be subclassified according to the rate at which they beat. Early models were set at a constant BPM rate, regardless of the kind of activity in which the wearer was engaged.

Today, pacemakers are either of the demand- or rate-responsive variety. Demand generators shut off when your heart beats normally. However, when heart rate falls below a specific number of BPM (usually 70 ), a special circuit automatically turns the pacemaker on.

Most pacemakers currently implanted are of the single-chambered, ventricular-demand type.

Stimulation and pacing occur in the ventricle.

Doctors find this model particularly attractive for less active persons.

Rate-responsive models feature varying rhythm degrees within a wide range of preset BPM. The sensing device responds to vibrations created by body movement. The vibrations are transmitted through bones, fluid, and body tissue as pressure waves. These, in turn, bend the titanium shield, creating an electric signal in the process. The more activity, the more the titanium bends, the stronger the impulses and the quicker the heart beats. Most rate- responsive generators are programmed to output between 60 to 150 BPM.

While most pacemakers are intended to control bradycardiac arrhythmias, at least one such device is used to treat tachycardiac patients. When the heart rate increases beyond the established limit, this pacemaker delivers stimuli based on the type of tachycardia involved. These artificial impulses collide with the heart's own signals, thereby canceling out each other. Normal heart rhythm then returns.

\section{Setting the pace}

Virtually all modern pacemakers are programmable to some degree. Your physician sets the limits based on any existing disease you may have, as well as what medication you may be taking. These same factors, along with your life-style, will determine what kind of pacemaker-if any-you should receive.

\section{Pacemaker protocol}

Living with a pacemaker should not stop you from your normal activities. Nevertheless, certain guidelines should be followed to maintain a healthy rhythm:

- Remember to take your pulse for one full minute at least once a day. (Ask your doctor for the acceptable beat range programmed for your particular pacemaker.) Record these readings and bring them to your next doctor's appointment.

- If you detect an occasional irregular rhythm, don't worry. Sometimes the artifical pacemaker competes with your natural one.

- During the first 2 months after the implant, avoid any sudden jerky movements. Don't over-reach. The device needs time to settle in place.

- Shortly after the implant, should your pulse suddenly drop below the 60 BPM, call your doctor immediately. A broken lead or other mechanical failure may exist. Remember, a substantially decreased heart rate robs your system of vital nutrients, including oxygen. Fainting, coma, or in severe instances, even death can ensue.

- Likewise, if your heart rate registers above 100 BPM, contact your physician. Left unchecked, this condition could lead to a serious situation in which the muscles lining the heart contract wildly out of control.

- Take medicine as prescribed.

- Exercise according to your doctor's advice, but don't overdo it.

- Don't be afraid to bathe and shower as usual. The generator is water-resistant. 
- Stay away from electric currents if possible. Metal detectors and microwaves (especially standing right in front of one) may create electric impulses that compete with your present pacemaker. Show your pacemaker identification card to airport personnel to avoid walking through metal detectors.

Remember to inform doctors and dentists you're wearing a pacemaker. Their equipment may also interfere with the electric signals.

- Keep all doctor's appointments so your pacemaker rate can be monitored, and if necessary,

reprogrammed. Regular checkups are particularly important at the beginning of the battery's life as well as near its end. Old batteries cannot be recharged. They must be surgically replaced.

Overall, pacemakers can improve the quality of life for appropriate patients. However, the decision to undergo this procedure should not be taken lightly. You shop around for the right mechanic to work on your car's body, so look for physician consensus when it comes to your own body work.

(All material in the Patient Health Guide is reviewed by JAOA physician referees.)

\section{References:}

A leap for hearts. By A.K. Kurtz. Health, vol 17, p 19, May 1985.

Antiarrhythmic therapy in the elderly: Pacemakers and drugs. By Neil D. Berman. Geriatrics, vol 41, pp 61-72, February 1986.

The Columbia University College of Physicians and Surgeons Complete Home Medical Guide. Edited by David F. Tapley, et al. Crown Publishers. Inc., New York, 1985

Device ups heart output. Science Digest, vol 90, p 92, September 1982.

Indications for and management of cardiac pacemakers. Edited by Nicholas J. Cotsonas, Jr, et al: Disease-a-Month, vol 31, pp 42, March 1985.

Living with your pacemaker. American Heart Association, Dallas, 1987.

Medical electronics improve quality of life for patients. By Brian J. Hogan. Design News, vol 42, pp 54-58, February 3, 1986.

Med Tech: The layperson's guide to today's medical miracles. By Lawrence Galton. Harper \& Row, Publishers, Inc., New York, 1985.

The Merck Manual of diagnosis and therapy. Edited by Robert Berkow et al. Ed. 15, Merck Sharp and Dohme Research Laboratories, Rahway, New lersey, 1987.

Pacemaker mimics healthy heart's beat. Design News, vol 42, pp 35-36, September 22, 1986.

Pacemakers: Normal function. By Scott R. Spielman. Geriatrics, vol 40 , pp 65-71, June 1985. 


\section{di STUART PHARMACEUTICALS}

1987 ICIAmericas Inc

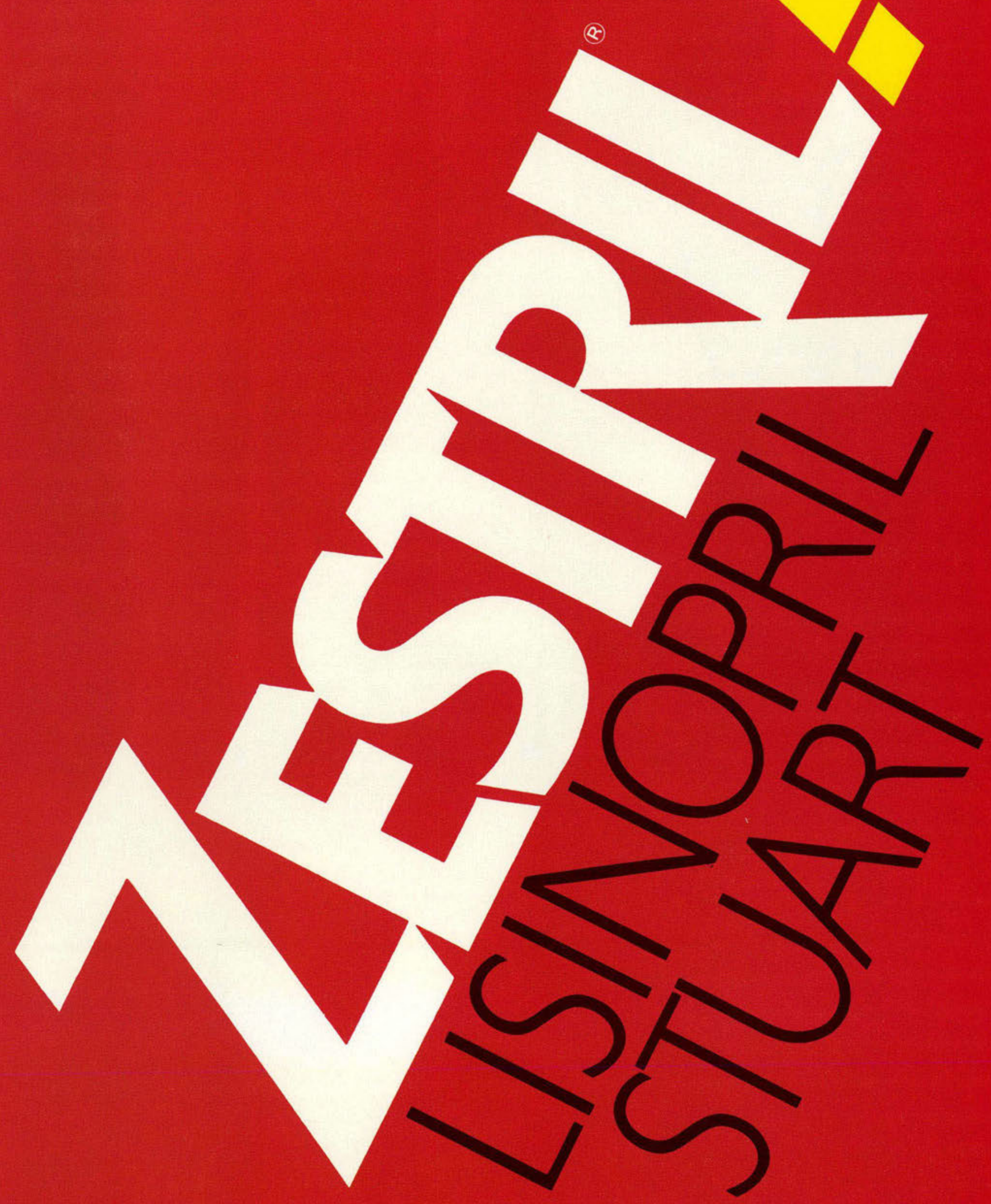




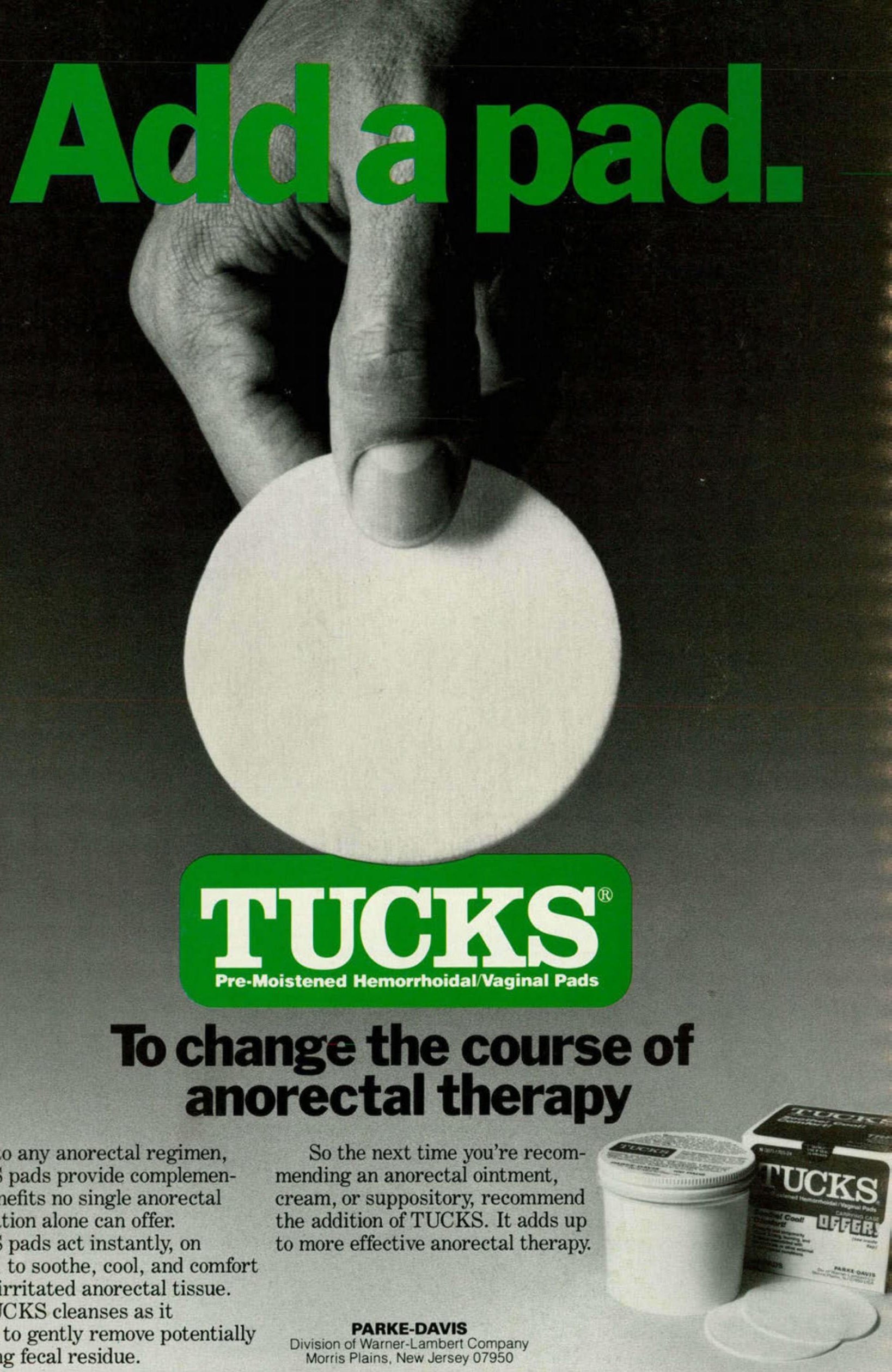

Added to any anorectal regimen, TUCKS pads provide complementary benefits no single anorectal preparation alone can offer. TUCKS pads act instantly, on contact, to soothe, cool, and comfort tender, irritated anorectal tissue. And TUCKS cleanses as it soothes to gently remove potentially irritating fecal residue.
So the next time you're recommending an anorectal ointment, cream, or suppository, recommend the addition of TUCKS. It adds up to more effective anorectal therapy. 